\title{
Transatlantica
}

Revue d'études américaines. American Studies Journal

\section{La politique du United States Forest Service}

Quel avenir pour les forêts publiques?

\section{Olivier Delbard}

\section{OpenEdition}

Journals

Édition électronique

URL : http://journals.openedition.org/transatlantica/568

DOI : $10.4000 /$ transatlantica.568

ISSN : $1765-2766$

Éditeur

AFEA

Référence électronique

Olivier Delbard, « La politique du United States Forest Service », Transatlantica [En ligne], 1 | 2005, mis en ligne le 24 mars 2006, consulté le 29 avril 2021. URL : http://journals.openedition.org/

transatlantica/568; DOI : https://doi.org/10.4000/transatlantica.568

Ce document a été généré automatiquement le 29 avril 2021.

\section{(c) ()}

Transatlantica - Revue d'études américaines est mis à disposition selon les termes de la licence Creative Commons Attribution - Pas d'Utilisation Commerciale - Pas de Modification 4.0 International. 


\title{
La politique du United States Forest Service
}

Quel avenir pour les forêts publiques?

\author{
Olivier Delbard
}

1 Le gouvernement fédéral possède et gère aujourd'hui près de $34 \%$ des forêts des États-Unis ; dans certains États de l'Ouest, les forêts fédérales couvrent jusqu'à $25 \%$ de la superficie totale. Les deux tiers de ces forêts fédérales (193 millions d'acres), représentant plus de 8,5\% de la superficie des Etats-Unis, sont gérés par le USDA Forest Service, puissante agence fédérale née en 1905 et forte aujourd'hui de plus de 30000 employés.

2 Dans le cadre du débat qui agite la société américaine sur la gestion des terres publiques aujourd'hui, la question de l'avenir du U.S.D.A. Forest Service est particulièrement pertinente. Il y a un siècle déjà, la création de l'agence s'était faite dans un contexte passionnel d'affrontements portant sur l'usage des forêts appartenant au domaine public.

3 L'objectif ici est de tenter de comprendre comment et pourquoi la politique du Forest Service donne lieu depuis au moins deux décennies à tant de critiques et de controverses, aussi bien en termes de gestion des ressources que, plus fondamentalement, de légitimité politique. Un rapide bilan historique de la politique menée par l'agence permettra dans un premier temps de mieux comprendre le clivage qui s'est installé durablement dans la société américaine à propos de l'« exploitation » des forêts publiques. L'histoire du Forest Service montre en effet que la controverse idéologique, très forte au moment de la fermeture de la Frontière, restera sous-jacente tout au long du développement de l'agence, alors même que cette dernière symbolise le triomphe de l'idéal progressiste et le productivisme au service de la Nation tout entière.

Le débat qui s'intensifie à partir de la fin des années soixante, marquées par la montée en puissance $\mathrm{du}$ mouvement écologiste dans la sphère politique, porte fondamentalement sur le dilemme suivant: s'agit-il de ne considérer les forêts publiques que d'un point de vue économique, c'est-à-dire générateur de revenus tirés directement de l'exploitation, ou ne faudrait-il pas au contraire envisager que ces 
terres ont une valeur intrinsèque, ce qui implique la nécessité de les protéger de tout développement pour des raisons éthiques? Cette priorité absolue donnée à la valeur éthique des terres publiques trouve son origine dans les écrits d'un directeur du Forest Service, Aldo Leopold ${ }^{1}$; c'est dans ce sens qu'il faut comprendre ici le conflit entre " économique » et « éthique ».

5 Le Forest Service est aujourd'hui plus que jamais sur la sellette : certains en demandent même l'abolition pure et simple, clamant haut et fort que l'idéal démocratique est perverti, que la mission de l'agence ne répond plus à la réalité des attentes de la société, ou encore que son fonctionnement serait le reflet de l'incompétence du pouvoir fédéral en matière de gestion publique (des terres en l'occurrence). Ces critiques mettent en lumière l'antagonisme régnant entre l'industrie (forestière, agricole et minière), ses relais institutionnels classiques au Congrès ou au sein de l'Exécutif, et l'écologie politique, mouvement en perpétuelle évolution et aux multiples ramifications tant aux niveaux local que national.

6 Cet antagonisme soulève certaines questions relatives aux principes mêmes de la nation américaine : le pouvoir fédéral, par le biais du Forest Service, a-t-il la légitimité de gérer cette part importante des terres du pays? Ne serait-ce pas aux États de prendre en charge la gestion des forêts publiques ? Y a-t-il d'ailleurs jamais eu un mandat clair et précis? Le concept même de «terre publique » dont la définition traditionnelle ${ }^{2}$ est «l'utilisation optimale des ressources pour le bien du plus grand nombre » reste-t-il valide dans un contexte d'affrontements partisans et dans une logique d'intérêts particuliers? Ne vaudrait-il d'ailleurs pas mieux privatiser ces terres par souci d'efficacité ?

7 Plus fondamentalement encore, le cas du Forest Service est le révélateur d'un débat politique, institutionnel et intellectuel depuis la fin de la Frontière qui dépasse largement le cadre des terres publiques. Cet affrontement entre deux conceptions diamétralement opposées des forêts fédérales offre un nouvel éclairage sur la vision même du processus historique américain et de ses valeurs fondatrices - ou, en d'autres termes, de l'inévitable choc entre l'utilitaire et l'imaginaire comme moteurs du rêve américain.

8 Enfin, il est clair que ce conflit entre l'éthique et l'économique est particulièrement sensible depuis le début des années quatre-vingt : l'administration Reagan, que l'on ne peut suspecter de sympathie pour l'écologie politique, privilégie de toute évidence le développement économique au détriment de la protection des ressources naturelles ${ }^{3}$. Vingt ans plus tard, les nominations aux postes clés pour l'environnement et la gestion des ressources naturelles de l'administration Bush font plus que jamais de cette question un enjeu majeur de sociétét.

La naissance des terres publiques

Bien que la création par Ulysses Grant du premier parc national à Yellowstone en 1872 marque les débuts officiels de la " mise à l'écart » d'espaces naturels aux États-Unis, ce n'est qu'en 1890 avec la fermeture officielle de la Frontière qu'apparaissent des législations fédérales destinées à préserver certaines portions de terres de l'immense domaine public de l'Ouest américain. En cette même année 1890, après une longue bataille menée avec acharnement et conviction par John Muir, le Congrès vote le Yosemite Act, que le président Harrison signe sans délai. La loi reconnaît la nécessité de préserver le wilderness, patrimoine historique d'une valeur inestimable. L'année suivante, en 1891, le Congrès vote le Forest Reserve Act, qui donne au président le 
pouvoir de prélever du domaine public certaines forêts pour créer des forest preserves (qui deviendront par la suite les national forests). Bien que la fonction de ces forêts ne soit pas clairement définie par la loi, cette première législation marque la volonté du gouvernement fédéral de contrôler directement de vastes étendues de forêts à l'Ouest. Pour le mouvement conservationniste, c'est une grande victoire, qui donne un cadre légal à la lutte contre l'exploitation excessive et désordonnée des ressources naturelles dans le grand Ouest américain.

10 Les années qui suivent seront marquées par un intense débat entre l'aile radicale du mouvement pour la défense des terres publiques (John Muir et les préservationnistes ${ }^{5}$ ) et les conservationnistes, partisans d'un compromis avec les industries forestière et minière, sans oublier l'opposition farouche des petits exploitants favorables à la privatisation des terres. Ainsi, les préservationnistes, sans s'opposer totalement à l'exploitation commerciale de la forêt, voient-ils d'emblée dans ces étendues boisées des zones de wilderness, dont la valeur est intrinsèquement liée à l'histoire et au caractère du peuple américain ${ }^{6}$.

Quoi qu'il en soit, le Forest Management Act, voté en 1897, marque une victoire très nette pour l'industrie forestière : en effet, il est clairement stipulé que le but recherché est de: «Improve the forest within the boundaries, or for the purpose of securing favorable conditions for water flows, and to furnish a continuous supply of timber for the use and the necessity of the citizens of the United States ${ }^{7}$. "

La conception strictement utilitariste, fortement influencée par les méthodes européennes de gestion forestière, a séduit les principaux artisans de la loi, car elle symbolise le progrès, par la mise en pratique de méthodes scientifiques, gages de gestion prudente et raisonnée (wise management) des ressources naturelles. Cette loi organique de $1897^{8}$ régira la politique des forêts nationales jusqu'en 1976. dans les années qui suivent le passage de la loi, pour deux raisons principales : l'une tient à la volonté personnelle des présidents qui se succèdent (Cleveland et surtout Roosevelt), l'autre est liée à l'accession des derniers territoires de l'Ouest à l'Union ; en effet, l'appropriation de terres publiques par le gouvernement fédéral est dans la plupart des cas une des conditions d'accession à l'Union pour ces territoires'.

Du progressisme au productivisme

14 Le président Roosevelt, si fier de sa politique de parcs nationaux et de refuges pour la faune et la flore sauvage, ami personnel de John Muir, institue le Forest Service non sans quelque réserve, mais poussé par Gifford Pinchot, forestier pour qui il a beaucoup d'estime et qu'il nomme à la tête de l'agence.

Dès sa naissance, la philosophie utilitariste et progressiste de l'agence est clairement affichée. Selon les propres termes de Gifford Pinchot, la mission de l'agence est de: " provide the greatest amount of good for the greatest amount of people in the long run $»^{10}$. Pinchot, forestier de profession rompu aux méthodes européennes, marquera durablement de son empreinte la philosophie de l'agence fédérale, fondée sur le progressisme scientifique du tournant du XIXe siècle ${ }^{11}$. Le Progressisme américain croit en effet en l'avènement de la connaissance scientifique comme moteur suprême de la démocratie américaine ${ }^{12}$. L'approche scientifique, objective et rationnelle, est perçue comme le meilleur mode de gouvernement puisqu'elle permet au plus grand nombre d'atteindre un bien-être matériel, ce qui signifie à long terme la fin de la misère et du manque de confort, qui va de pair avec un nouveau souffle spirituel. Il s'agit bien, pour 
reprendre les termes de Samuel Hays d'un "évangile de l'efficacité " (gospel of efficiency). Ainsi le mouvement conservationniste modéré voit-il dans la politique fédérale des terres publiques le meilleur moyen de répondre aux besoins de la population dans la durée par une utilisation la plus efficace possible de ressources telles que le bois, les pâturages ${ }^{13}$ ou les minerais ${ }^{14}$. Les forêts nationales sont le parfait reflet de l'idéal démocratique puisqu'elle sont gérées pour le bien du peuple américain tout entier et que l'objectivité scientifique est la meilleure garantie démocratique contre les intérêts partisans, quels qu'ils soient. D'où le rattachement du seul Forest Service au Département de l'Agriculture, plus « utilitaire » et moins politique que le Département de l'Intérieur ${ }^{15}$.

Fort logiquement, l'agence développe une politique d'optimisation des ressources de la forêt, principalement par la coupe du bois, mais aussi en ouvrant certaines terres à l'exploitation minière et en accordant des permis de pâturage aux ranchers de l'Ouest. Le système mis en place semble équitable et équilibré puisque les revenus reviennent directement au gouvernement fédéral (de façon directe) et aux États de l'Ouest (principalement) auxquels le Forest Service reverse une redevance destinée à la construction de routes et d'écoles. Pendant la Grande Dépression, le Forest Service joue un rôle stratégique auprès de la population en offrant de nombreux emplois aux chômeurs (principalement dans l'Ouest et le Nord-Ouest). Puis l'agence contribuera de manière significative à l'effort de guerre en fournissant à la nation bois et ressources stratégiques.

Les années d'après-guerre marquent un tournant incontestable : la production de bois est doublée en l'espace de quelques années, l'industrie forestière adopte des méthodes modernes et intensives, répondant par là même aux besoins considérables en bois de l'Amérique des années cinquante. Ainsi, de sa naissance jusqu'à cette période de l'après-guerre, la mission du Forest Service est acceptée de façon quasi-unanime et le progressisme scientifique semble avoir fait ses preuves, même si un de ses éminents représentants, Aldo Leopold, commence à essaimer dans les esprits, en arguant que c'est l'éthique qui doit à terme l'emporter sur l'économique dans la gestion de la terre ${ }^{16} \mathrm{~L}$ a publication des écrits de Leopold, dans les années quarante, coïncide d'ailleurs avec l'apparition d'une nouvelle utilisation des forêts nationales: outre les grands «monuments" nationaux du National Parks Service et les refuges pour la faune et la flore sauvages gérés par le Fish and Wildlife Service, les forêts fédérales deviennent pour un nombre croissant de citoyens des lieux de loisirs (randonnée, chasse, pêche, camping etc.). La dimension utilitaire des forêts nationales se trouve ainsi confrontée à des valeurs non strictement économiques, qui se font l'écho de l'esprit pionnier, dont les traits furent glorifiés par Turner au moment de la fermeture de la Frontière.

Luttes et litiges

Cette coexistence entre valeurs marchandes et non-marchandes est entérinée par le Multiple Use Sustained Yield Act de 1960. Cette loi, tout en reconnaissant les multiples utilisations possibles des ressources naturelles (incluant les loisirs, la préservation de la faune et de la flore) et engageant le Forest Service à diversifier ses activités dans ce but, donne cependant une place prépondérante à l'exploitation économique des forêts, restant donc largement favorable aux intérêts du lobby forestier. Il est clair que le Forest Service reste, par le biais du système des redevances versées aux États ${ }^{17}$, étroitement soumis aux intérêts de l'industrie forestière. En témoigne d'ailleurs la production de bois qui augmente sans cesse pour atteindre un niveau record en 1973. 
Cette période, marquée par la destruction quasi-totale des forêts primaires de l'Ouest, entraîne inévitablement l'émergence d'un contre-pouvoir influent issu des rangs de grandes associations de protection de l'environnement, à l'image du Sierra Club qui retrouve le terrain de l'activisme politique et commence à exercer une forte pression sur le Congrès pour arrêter le massacre des forêts anciennes.

Cette émergence d'une force environnementale sur la scène politique nationale conduit le Congrès à voter deux grandes lois de protection du patrimoine naturel national. C'est d'abord le Wilderness Act de 1964 qui institue la création de zones de wilderness, protégées de toute exploitation humaine directe. Le Forest Service, malgré son opposition, doit se résoudre à "abandonner " environ un cinquième des forêts fédérales. Par ailleurs, la création d'une agence fédérale chargée de la protection de l'environnement, l'Environmental Protection Agency en 1972 et le passage du Endangered Species Act en 1973 sont autant de contraintes légales imposées à l'agence.

Cette dernière, soumise à de fortes dissensions internes, tente de concilier les intérêts de plus en plus divergents qui apparaissent dans la société en s'appuyant sur le National Environment Policy Act de 1969, dont l'objectif principal est d'associer le public aux politiques environnementales de la nation. La loi ouvre la porte aux audiences publiques qui se multiplient, ainsi qu'aux actions en justice, qui en sont le corollaire. Le Forest Service, dont la légitimité, aussi bien politique que scientifique, est sérieusement ébranlée, se trouve alors entraîné dans une logique d'affrontements partisans, aussi bien à Washington qu'au niveau local. Le nombre d'actions en justice et d'audiences publiques est tel que l'agence est vite engluée dans un fonctionnement de plus en plus coûteux, complexe et lourd. Chaque nouveau plan de gestion d'une forêt nationale implique une concertation difficile et longue avec les groupes d'intérêts locaux, une collaboration problématique avec toutes les agences fédérales concernées et le respect d'une législation complexe et parfois contradictoire.

21 Le plus bel exemple du fossé qui se creuse inexorablement entre le Forest Service et une part sans cesse croissante de l'opinion publique est l'arrêt Monongahela de 1973 (West Virginia Division of the Izaak Walton League of America, Inc. vs Butz ${ }^{18}$ ), qui, sur la base d'une interprétation de la Loi organique de 1897, déclare illégale les coupes rases (clearcutting) dans les forêts nationales; l'arrêt sera confirmé en appel en $1975^{19}$. Suivie d'actions intentées un peu partout dans le pays, cette décision est un coup terrible pour le Forest Service dont le productivisme scientifique est fondamentalement remise en cause.

Face à cette situation, le Forest Service presse le Congrès d'adopter une nouvelle législation : ce sera en 1976 le National Forest Management Act qui remplace la loi de 1897. La loi tente de concilier les intérêts divergents de l'industrie et des défenseurs de l'environnement en couplant le principe d'usage multiple à celui de gestion durable de la forêt, l'objectif central étant «the greatest net public benefit »" ${ }^{20}$. En tentant de synthétiser tous les intérêts catégoriels, la loi cherche à restaurer la confiance de l'opinion et donc rendre à l'agence sa légitimité démocratique. Le Congrès tente dans les faits de résoudre un conflit idéologique finalement assez proche de celui qui agitait l'Amérique de John Muir et de Gifford Pinchot au tournant du XIXe siècle ${ }^{21}$.

23 L'effet à court terme de la loi semble bénéfique aux défenseurs de l'environnement; la production de bois chute en effet jusqu'en 1983 (restant cependant au niveau des années soixante) ${ }^{22}$, mais l'atmosphère partisane des années Reagan rétablit l'industrie forestière dans son plein droit: la production s'accroît à nouveau pour atteindre un record absolu en 1989. Les années quatre-vingt bousculent le débat traditionnel : tandis 
que l'opinion s'émeut des destructions massives des forêts du Nord-Ouest pacifique, les grandes associations écologistes, prises au piège de l'institutionnalisation à Washington, sont dépassées par de nouvelles organisations radicales et de terrain ${ }^{23}$. L'appareil législatif est suffisamment flou et incohérent pour favoriser la grande industrie et les États de l'Ouest font aisément valoir leurs intérêts au niveau fédéral. Les militants écologistes sont eux-mêmes divisés sur la question de l'usage des forêts nationales ; pour certains, les activités de loisirs sont aussi nuisibles à l'environnement que la coupe des arbres. Les années quatre-vingt marquent de fait une polarisation accrue, due en grande partie à une intense couverture médiatique ainsi qu'à l'apparition de nouvelles lignes de partage politiques, dont celle entre les intérêts locaux et nationaux. Se forment d'ailleurs des mouvements d'initiatives locales, les watershed initiatives, incluant tous les groupes d'intérêts concernés qui élaborent ensemble des propositions en toute indépendance, témoignant par là même de l'impuissance de Washington en la matière.

La déforestation massive de la fin des années quatre-vingt dans l'Ouest et le Nord-Ouest est brutalement interrompue par la controverse autour de la protection de la chouette tachetée (spotted owl ${ }^{24}$ ) qui aboutit finalement à un quasi-arrêt des coupes dans le Nord-Ouest. Entre 1989 et 1998, les ventes de bois des forêts nationales chutent de $70 \%{ }^{25}$. Cet effondrement de la production au cours des années quatre-vingt-dix remet en cause la mission même de l'agence, en niant sa politique scientifico-utilitariste.

Du productivisme à l'éthique?

Ce tournant idéologique est entériné par l'administration Clinton qui, dès 1992, renouvelle en profondeur le personnel du Forest Service, donnant la priorité aux écologues plutôt qu'aux forestiers. À partir de 1992, le discours s'articule autour de nouveaux concepts: le "management des écosystèmes " (ecosystem management,) qui substitue à l'approche rationnelle classique objective une logique dynamique, relationnelle et morale, et le principe éthique de stewardship, qui insiste sur le respect de l'intégrité de la terre, en tentant de réunir utilitarisme et préservationnisme en une approche durable de l'environnement. Les propos $^{26}$ de Mike Dombeck, directeur du Forest Service jusqu'en avril 2001, illustrent bien cette volonté de cohérence retrouvée. Ainsi, tout en n'abandonnant pas totalement l'approche utilitariste ("we have used the land to build our homes and to feed and clothe our people »1), il met indéniablement l'accent sur la valeur intrinsèquement éthique des terres publiques, en redonnant à la politique fédérale une légitimité historique fondatrice, celle de la préservation d'un patrimoine commun, vestige matériel de l'esprit pionnier qui a formé le caractère national :

As citizens of the United States, each of us owns a share of millions of acres of land. This is our birthright, the bequest of our forebears, many of whom died securing the land and the freedom we enjoy, ( $\mathrm{p} 1$ )

et plus loin:

Conservation is a uniquely American idea born of our uniquely American heritage. Europe has its great castles and works of art, Africa its ancient pyramids and cultures. Here in America we have our wild places, the first homes of the American Indians. These untamed landscapes are what remain of the pioneer spirit that shaped this nation's character. (2) de son courant de pensée (selon lequel les valeurs de l'esprit pionnier, constitutives du caractère national, sont la marque de supériorité du modèle démocratique américain) 
est loin de faire l'unanimité. Comment en effet privilégier un usage non productif des forêts publiques tout en maintenant le même type de fonctionnement économique et institutionnel ? Cette attitude ambiguë de l'administration Clinton va de fait favoriser l'éclosion d'un "écologisme ultra-libéral» (free market environmentalism), avatar du néo-conservatisme né pendant les années soixante-dix ${ }^{27}$, très présent dans les départements de sciences politiques et dans les médias. Ainsi, Robert Nelson, professeur de sciences politiques à l'université du Maryland, dans un ouvrage récent, développe une série d'arguments contre le Forest Service et la politique fédérale de gestion des forêts publiques.

La thèse de Nelson part d'un constat : la politique fédérale des forêts est un fiasco, que ce soit aux plans économique, scientifique ou institutionnel. L'effondrement des ventes de bois n'a pas modifié les besoins du public; les importations en provenance du Canada ont dû être presque doublées pendant la dernière décennie, la population américaine consommant plus de $25 \%$ de la production mondiale. En outre, cet effondrement de la production représente une perte de revenus considérable pour les États de l'Ouest, dépendants de la redevance fédérale. L'incohérence du processus politique associé au montage du budget annuel de l'agence, la vente du bois à un prix inférieur à celui du marché en sont d'autres exemples. Par ailleurs, le management scientifique est un échec, comme le prouvent l'érosion des sols, les coupes rases ou encore les feux de forêts des années quatre-vingt-dix. Pour Nelson, cette approche scientifique a toujours été porteuse de tensions entre les idéaux technocratiques et le fonctionnement de la démocratie américaine. En d'autres termes, la caution scientifique est un leurre qui a vidé le Forest Service de toute légitimité démocratique. Le pouvoir fédéral, tiraillé entre des intérêts contradictoires et finalement convergents d'un point de vue objectif, n'est pas à même de gérer les forêts publiques avec efficacité. Les États de l'Ouest, tributaires des redevances fédérales, ne sont pas traités démocratiquement, et ne peuvent faire entendre leur voix qu'au Sénat. Nelson est donc fort logiquement partisan d'une privatisation partielle et d'un transfert de compétence vers les États (ce qui, selon lui, rétablira le fédéralisme malmené dans cette affaire). Il applique le principe néo-libéral selon lequel seul le marché est la réponse appropriée à une politique efficace de l'environnement et des ressources naturelles. Mais il se fait également porte-parole d'intérêts partisans, ceux des Républicains, de l'Ouest notamment ${ }^{28}$.

Pour les partisans d'une approche néo-libérale de l'environnement, la gestion fédérale des forêts publiques ne fut qu'une étape historique liée à l'intégration de l'Ouest à l'Union. Cet héritage culturel symbolique n'a aucune valeur aujourd'hui, car il ne repose sur aucune rationalité économique. Si la plupart des critiques de Nelson sont pertinentes et mettent en lumière toute l'ambiguïté de la politique des terres publiques, ses propositions s'attaquent dangereusement au principe même d'espace public dans la Nation en niant la nécessité d'une politique de préservation d'un espace légitimé par l'Histoire. De ce point de vue, le processus historique de développement repose sur un transfert inéluctable de l'exercice du pouvoir démocratique vers le local et le privé. Cette interprétation du progrès a beau jeu de diaboliser les partisans d'une mise à l'écart des forêts fédérales, en y voyant le rejet radical de la civilisation même. S'il est vrai qu'une politique préservationniste remet d'une certaine façon en cause la logique ethnocentrique, productiviste et utilitariste des États-Unis, elle est aussi l'expression d'une volonté profondément ancrée dans l'histoire, en ce qu'elle est 
intimement liée à la constitution de l'identité américaine ${ }^{29}$. La question des forêts nationales est donc un excellent révélateur du conflit inhérent au processus historique américain : peut-on et doit-on assigner à ces terres publiques une valeur éthique, intrinsèque, absolue, au-delà de l'évidence économique ? Dans son essai paru en 1949, The Land Ethic, Leopold avance l'idée audacieuse que l'être humain doit développer une " conscience intense de la terre» ( intense consciousness of land ${ }^{30}$ ) pour enfin réaliser qu'il est un élément d'une communauté complexe et interdépendante d'humains et de non-humains ( $a$ member of a community of interdependent parts » ${ }^{31}$ ); c'est en prenant conscience de cette dimension éthique qu'il pourra se comporter en véritable citoyen :

A land ethic changes the role of Homo Sapiens from conqueror of the land-community to plain member and citizen of it. It implies respect for his

fellow-members, and also respect for the community as such ${ }^{32}$.

Cette éthique est par essence politique, car elle marque l'aboutissement de l'idéal communautaire démocratique, dans lequel en fin de compte les éléments non-humains sont reconnus en droit. Cependant, alors même que l'écologisme néo-libéral semble éminemment dangereux, on imagine mal le gouvernement fédéral adopter le «biocentrisme holistique ${ }^{33}$ » de Leopold et tourner le dos au paradigme dualiste moderne.

Les tribunaux en dernier recours?

31 Le pouvoir judiciaire serait-il alors la seule voie pour sortir la nation de cette impasse politique et idéologique ? Ce dernier, si l'on en juge à l'aune d'arrêts récents, ne semble pas apporter de réponse satisfaisante : en 1998, dans l'affaire Ohio Forestry Association vs Sierra Club ${ }^{34}$, la Cour d'appel pour le sixième Circuit a dû examiner la validité du plan d'exploitation de la forêt nationale de Wayne dans l'ohio. Pour le Sierra Club, ce plan "permits too much logging and too much clearcutting ". Après examen de la requête de l'organisation écologiste, la Cour juge le plan d'exploitation légal: "the Plan does not inflict significant practical harm upon the interests that the Sierra Club advances »; pour la Cour, les arguments présentés par le Sierra Club ne sont pas encore " mûrs » (la ripeness doctrine est appliquée); tout en reconnaissant le fait que ses arguments seront sans doute recevables par la suite ( The Sierra Club thus will have ample opportunity later to bring its legal challenge at a time when harm is more imminent and more certain »), l'appel est rejeté. Cette décision, qui reconnaît implicitement le bon droit des défenseurs de l'environnement, montre clairement que c'est au politique de tenter de résoudre ce conflit d'intérêts permanent concernant les forêts publiques.

S'il est difficile aujourd'hui d'augurer de l'avenir des forêts nationales, la nomination en 2001 de Dale Bosworth, forestier de profession, à la tête du Forest Service indique logiquement le choix pour l'administration Bush du retour à un productivisme utilitaire "sans valeur éthique ajoutée ", pour ne pas dire plus. L'histoire du Forest Service est la preuve que la question de l'utilisation de la forêt publique, substance éminemment politique, aura du mal à être résolue. Face à l'intensification de ce conflit d'intérêts, c'est aux citoyens de faire entendre leur voix : après tout, ces forêts leur appartiennent. 


\section{BIBLIOGRAPHIE}

Callicott, J. Baird. In Defense of the Land Ethic. Essays in Environmental Philosophy. Albany : State University of New York Press, 1989.

Clary, David. Timber and the Forest Service. Lawrence, Kansas : University Press of Kansas, 1985. Frome, Michael. Whose Woods These Are : The Story of National Forests. Garden City, N.Y., 1962. Hays, Samuel P. Conservation and the Gospel of Efficiency : the Progressive Conservation Movement, 1890-1920. Cambridge : Harvard University Press, 1959.

Leopold, Aldo. « The Land Ethic » in A Sand County Almanac with Essays on Conservation from Round River. New York : Ballantine Books, 1966 [Oxford University Press, 1949].

Nash, Roderick Frazier. Wilderness and the American Mind. Yale University Press, Third Edition, 1982.

Nash, Roderick Frazier. The Rights of Nature. A History of Environmental Ethics. The University of Wisconsin Press, 1989.

Nelson, Robert H. A Burning Issue. A Case for abolishing the U.S. Forest Service. Political Economy Forum, Rowman \& Littlefield Publishers, Inc, Lanham, 2000.

O'toole, Randal. Reforming the Forest Service. Washington D.C. : Island Press, 1988.

Sedjo, Roger A., éd. A Vision for the U.S. Forest Service. Goals for its next century. Resources for the Future, Washington D.C., 2000.

Turner, Frederick Jackson. The Frontier in American History. New York, 1920.

Worster, Donald. Nature's Economy : A History of Ecological Ideas, New York : Cambridge University Press, 1977.

Yaffee, Steven L. The Wisdom of the Spotted Owl : Policy Lessons for a New Century. Washington D.C. : Island Press, 1994.

\section{NOTES}

1. Voir Aldo Leopold, «The Land Ethic » in A Sand County Almanac with Essays on Conservation from Round River (New York : Ballantine Books, 1966 [First Edition : Oxford University Press, 1949]).

2. Pinchot, « to provide the greatest amount of good for the greatest amount of people in the long run ", définition donnée par le premier directeur du USDA Forest Service lors de sa création en 1905.

3. On pense aux forts remous occasionnés par les décisions prises par James $G$. Watt, à l'Intérieur, et Anne M. Gorsuch, à la tête de l'EPA (agence fédérale chargée de l'environnement, créée en 1972) pendant les trois premières années de l'administration Reagan. Ainsi, le budget de l'agence sera réduit de manière drastique : moins $30 \%$ en moyenne pour les années 1980-84 par rapport à 1979), plus de 200 postes d'experts supprimés, le nombre total d'employés en chute de plus de $25 \%$ en trois ans etc.; ajoutons à cela les déclarations à l'emporte-pièce de MM. Reagan et Watt, telles que « $80 \%$ de la pollution est due aux fleurs et aux plantes » (80\% of our pollution is caused by 
flowers and plants), discours prononcé le 16 septembre 1980, 1992 Earth Journal (Boulder, Colorado : Buzzworm Books, 1991), 161.

4. Christine T. Whitman arrive à la tête de l'EPA en 2001 avec un bilan mitigé en tant que gouverneur du New Jersey. Après deux années de mandat pour le moins agitées, c'est le très controversé gouverneur de l'Utah Mike Leavitt qui lui succède en novembre 2003 (il a quitté l'agence en décembre 2004). Ann Veneman nommée à l'agriculture est encore plus critiquée, du fait notamment de ses liens très étroits avec le lobby des géants de l'agro-alimentaire. Elle est remplacée en janvier 2005 par Mike Johanns. Quant au choix de Dale Bosworth en 2001 pour prendre la tête du Forest Service, il va clairement dans le sens des intérêts du lobby forestier (voir infra).

5. Un schisme apparaît en effet très rapidement au sein du mouvement pour la conservation des terres publiques entre les « préservationnistes » qui refusent la conception utilitariste et mettent en avant la défense d'une nature non «altérée » par l'action humaine, et les « conservationnistes » au contraire qui insistent sur l'utilisation raisonnée et prudente de ces mêmes terres (Voir Nash, op.cit., p 129).

6. Ils sont en cela les héritiers de " pionniers romantiques » tels que le peintre George Catlin (1796-1872) défenseur de la cause indienne, l'explorateur et homme politique John Wesley Powell (1834-1902) ou encore l'historien et explorateur de l'Oregon Trail Francis Parkman (1823-1893). Voir G. Catlin, Letters and Notes on the North American Indians (Grammercy Books, 1995) ; W.Debuys (ed.), The Essential John Wesley Powell (Island Press, 2004) ; F. Parkman, The Oregon Trail (Kessinger Publishing, 2004).

7. In General Appropriations Act of June 4, 1897, Chap. 2, 30 Stat. 34.

8. Complétée par le Weeks Act, voté en 1911, qui assigne le même objectif aux forêts fédérales de l'Est (Act of March 1, 1911 (36 Stat. 961).)

9. Avec en contrepartie la constitution de state lands.

10. Site Internet du USDA Forest Service, http ://www.fs.fed.us.

11. Pinchot dirigera le Forest Service de 1905 à sa création jusqu'en 1910, quand le président Taft, peu enclin à ses thèses, le renvoie avec pertes et fracas.

12. Toutes les grandes associations professionnelles naissent d'ailleurs pendant ces années-là, les forestiers créant leur propre organisme en 1900.

13. Dès la naissance du Forest Service, les propriétaires privés de bétail ont eu accès, par l'octroi de permis de pâturage, à certaines parcelles de terre. Cette pratique traditionnelle sur les terres publiques est largement critiquée aujourd'hui, car peu rentable.

14. Principalement sur les terres du Bureau of Land Management.

15. Contrairement à toutes les autres agences de gestion des terres publiques (Bureau of Land Management, National Parks Service et Fish and Wildlife Service) rattachées au Département de l'Intérieur.

16. Aldo Leopold (1887-1948) est sans conteste un des pères fondateurs de l'écologie politique contemporaine aux Etats-Unis, notamment dans le domaine de la gestion de la faune et de la flore en milieux naturels protégés. Scientifique formé à la sylviculture à Yale, il travaille pour le Forest Service de 1909 à 1928, puis occupe diverses chaires universitaires. La plupart de ses écrits seront regroupés juste après sa mort en 1949 sous le titre A Sand County Almanac, aujourd'hui un des grands classiques de la pensée écologiste. C'est dans ce livre qu'est pleinement développé et articulé le concept central de land ethic qui redéfinit radicalement la relation de l'homme à son environnement naturel (voir infra).

17. Redevances qui sont calculées sur la base des coupes effectuées. 
18. 367F. Supp 422 (N.D.W.Va. Nov 6 1973). La rivière Monongahela a donné son nom à une forêt nationale située en Virginie Occidentale.

19.522F. 2d945 (4th Cir. 1975).

20. National Forest Management Act, Amendment to The Organic Act, Sec. 14, http :// www.fs.fed.us/forum/nepa/nfmalaw.html

21. En effet, tout en étant scandalisé par l'exploitation des ressources naturelles, John Muir ne s'est jamais déclaré contre toute gestion de la forêt ; au contraire, il pensait qu'une exploitation prudente et limitée de certains domaines serait le meilleur gage de protection de vastes espaces naturels.

22. « Forest Service Timber Sales, 1905-95 », USDA Forest Service Cut \& Sold Reports, cité par Nelson, 60.

23. A l'image de Earth First! créée par le dissident Dave Foreman en 1978.

24. Ce dossier concerne la protection d'une espèce, le spotted owl, présente dans les forêts primaires du Nord-Ouest pacifique. Après d'interminables batailles juridiques et médiatiques, tant au niveau local que national, pendant plus d'une décennie, l'espèce est finalement reconnue « menacée » en 1992 (dans le cadre du Endangered Species Act de 1973), ce qui contraint le Forest Service et le Bureau of Land Management à geler l'activité sur de très nombreuses parcelles et donc à quasiment stopper l'extraction de bois.

25. U.S. Forest Service, Department of Agriculture, National Summary : Forest Management Program Annual Report, Fiscal Year 1997 (Washington, D.C.: U.S. Forest Service, July 1998) cité par Nelson, 152.

26. Conférence donnée à Duke University le 8 janvier 2001. http ://www.fs.fed.us/intro/speech/ jan08-Dombeck-conservation-investements-for-future-generations.htm.

27. Dans le sillage des mouvements dits de sagebrush rebellion par lesquels les petits propriétaires privés de l'Ouest cherchaient à obtenir le contrôle des terres publiques au niveau local.

28. Sa logique fait en effet la part belle à l'industrie et aux communautés rurales qui en dépendent, tout comme Clinton favorisait son électorat urbain de classe moyenne, sensible à la défense de l'environnement et grand utilisateur d'espace sauvages. Voir notamment Nelson, pp. 181-186.

29. Voir Nash, Wilderness and the American Mind, notamment les chapitres 6 (pp. 96-107) et 12 (pp. 200-237).

30. Ibid., 261.

31. Ibid., 239.

32. Ibid., 240.

33. Leopold est sans conteste le fondateur de cette théorie de l'écologie politique selon laquelle l'être humain n'est qu'un élément parmi d'autres d'une communauté interdépendante d'humains et non-humains. Le biocentrisme s'oppose donc à l'anthropocentrisme de la modernité occidentale. Quant au terme écocentrisme, il est généralement employé pour décrire les théories écologistes les plus extrêmes qui attachent autant, sinon plus de valeur à une bactérie rare qu'à un être humain par exemple. Voir à ce sujet Peter Hay, Main Currents in Western Environmental Thought, (Bloomington : Indiana University Press, 2002).

34. Ohio Forestry Association, Inc., petitioner v. Sierra Club et al., $\mathrm{N}^{\circ}$ 97-16, 105F.3D248. Consultable sur le site http ://www.supct.law.cornell.edu/supct 